ARTICLE

\title{
Identification of fidelity-governing factors in human recombinases DMC1 and RAD51 from cryo-EM structures
}

Shih-Chi Luo ${ }^{1,4}$, Hsin-Yi Yeh ${ }^{2,4}$, Wei-Hsuan Lan ${ }^{3}$, Yi-Min Wu', Cheng-Han Yang (iD ${ }^{1}$, Hao-Yen Chang ${ }^{2}$,

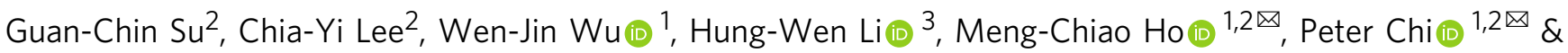
Ming-Daw Tsai (iD 1,2四

Both high-fidelity and mismatch-tolerant recombination, catalyzed by RAD51 and DMC1 recombinases, respectively, are indispensable for genomic integrity. Here, we use cryo-EM, $M D$ simulation and functional analysis to elucidate the structural basis for the mismatch tolerance of DMC1. Structural analysis of DMC1 presynaptic and postsynaptic complexes suggested that the lineage-specific Loop $1 \mathrm{Gln} 244$ (Met243 in RAD51) may help stabilize DNA backbone, whereas Loop 2 Pro274 and Gly275 (Val273/Asp274 in RAD51) may provide an open "triplet gate" for mismatch tolerance. In support, DMC1-Q244M displayed marked increase in DNA dynamics, leading to unobservable DNA map. MD simulation showed highly dispersive mismatched DNA ensemble in RAD51 but well-converged DNA in DMC1 and RAD51-V273P/D274G. Replacing Loop 1 or Loop 2 residues in DMC1 with RAD51 counterparts enhanced DMC1 fidelity, while reciprocal mutations in RAD51 attenuated its fidelity. Our results show that three Loop 1/Loop 2 residues jointly enact contrasting fidelities of DNA recombinases.

\footnotetext{
${ }^{1}$ Institute of Biological Chemistry, Academia Sinica, Taipei, Taiwan. ${ }^{2}$ Institute of Biochemical Sciences, National Taiwan University, Taipei, Taiwan. ${ }^{3}$ Department of Chemistry, National Taiwan University, Taipei, Taiwan. ${ }^{4}$ These authors contributed equally: Shih-Chi Luo, Hsin-Yi Yeh. ${ }^{凶}$ email: joeho@gate. sinica.edu.tw; peterhchi@ntu.edu.tw; mdtsai@gate.sinica.edu.tw
} 
$\mathrm{H}$ omologous recombination (HR) is a well-conserved cellular process for the repair of DNA double-strand breaks $^{1-4}$, the rescue of stalled/collapsed replication forks $^{2,5}$, and meiosis ${ }^{6-8}$. During HR, a recombinase binds to single-stranded DNA (ssDNA) and forms a right-handed helical nucleoprotein filament, known as a presynaptic filament, which then searches double-stranded DNA (dsDNA) for homology. Upon encountering a homologous sequence, the presynaptic filament pairs with the complementary strand, resulting in the displacement of the non-complementary strand from the duplex to generate a displacement loop structure and promote DNA strand exchange ${ }^{9-12}$.

Eukaryotic RAD51 and DMC1 both catalyze strand displacement and strand exchange. Interestingly, DMC1 possesses the ability of stabilizing mismatch-containing recombination intermediates (low-fidelity recombinase), whereas the RAD51 lacks the ability to do so (high-fidelity recombinase) ${ }^{13-17}$. RAD51 and DMC1 are members of the RecA family of DNA recombinases and share $\sim 50 \%$ amino acid identity and similar biochemical properties ${ }^{6,18-21}$. Both are ATP-dependent DNA-binding proteins that engage DNA in nucleotide triplet clusters and extend the DNA to $\sim 1.5$-fold $^{18,22-25}$. The bound DNA is organized into near B-form base triplets separated by $\sim 8 \AA$ between adjacent triplets ${ }^{26}$. The strand invasion products mediated by RAD51 and DMC1 filaments are both stabilized in three-nucleotide steps ${ }^{27,28}$. Interestingly, single-molecule studies also showed that DMC1 can stabilize single, double, or triple mismatches within a triplet in heteroduplex DNA joints, whereas RAD51 cannot ${ }^{27,29,30}$. It has been suggested that DNA-binding loop 1 (Loop 1) and loop 2 (Loop 2) of recombinases harbor specifically conserved amino acids within either the RAD51 lineage or the DMC1 lineage $17,26,31-34$. Recently, the cryogenic electron microscopy (cryo-EM) structures of human RAD51 complexes with an ssDNA (presynaptic) and a dsDNA (postsynaptic) were reported, which provided additional insight in the mechanism of strand exchange $^{28,35}$. However, as there are eight lineage-specific residues in Loop 1 and Loop 2 (Fig. 1a) ${ }^{17}$, and as no structure of DMC1-DNA filaments is available, the structural basis of the contrasting fidelity between DMC1 and RAD51 remains a major puzzle.

In this work we use cryo-EM, molecular dynamics (MD) simulations, and two independent functional assays to tackle these problems. Our results lead to a dynamics-based mechanism for recombination fidelity that involves two controlling factors: a gate for the basepair triplet and a support for the DNA backbone. A tight gate and a loose backbone support contribute to the high fidelity of RAD51, whereas a loose gate and a tight backbone support contribute to the mismatch tolerance of DMC1.

\section{Results}

Cryo-EM structures of DMC1 presynaptic and postsynaptic filaments. We first solved the cryo-EM structures of human DMC1 filaments with ssDNA and dsDNA (homologous) in presynaptic and postsynaptic states (Structure 1, $3.33 \AA$ and Structure 2, $3.47 \AA$, respectively), in the presence of adenylylimidodiphosphate (AMP-PNP) and $\mathrm{Ca}^{2+}$. The detailed procedures of these two and three other structures addressed later are described in "Methods," whereas the workflows and original images and maps are shown in Supplementary Fig. 1 for all five filament structures. The parameters related to helical reconstructions and the detailed data collection, refinement, and validation statistics are listed in Supplementary Table 1. Similar to the corresponding complexes of RAD51 28,35 , both structures adopt a well-ordered helical structure, with six protein protomers interacting with six triplets of DNA (Supplementary Fig. 2).
Detailed structures of these two DMC1 filaments and their comparisons with the corresponding human RAD51 complexes $^{28}$ and other recombinases are shown in Supplementary Fig. 3 (protomer interfaces) and Supplementary Fig. 4 (ATP-binding site). Here we focus on the identification of factors responsible for the mismatch tolerance (low fidelity) of DMC1 and the contrasting high fidelity of RAD51.

Structure-based identification of fidelity-governing residues. Recently, Greene and colleagues ${ }^{17}$ reported that the difference between the fidelity of Saccharomyces cerevisiae (Sc) Dmcl and Rad51 can be attributed to three lineage-specific residues of Loop 1, corresponding to human DMC1 E241/Q244/K245 and RAD51 A240/M243/H244, respectively, based on functional and biophysical analyses of a variety of variants. Figure $1 \mathrm{~b}, \mathrm{c}$ show the comparison between the structures of ssDNA in the presynaptic complexes of human DMC1 and RAD51, whereas Fig. 1d-f show the same comparison for dsDNA in the postsynaptic complexes. Interestingly, of the eight lineage-specific residues, only three (red asterisks in Fig. 1a) are located in the close proximity of DNA: Q244/P274/G275 of DMC1 (corresponding to M243/V273/D274 of RAD51) and they show different structural properties. The sidechain of Loop 1 residue Gln244 (polar) in DMC1 points toward the backbone of the dsDNA, whereas the corresponding Met243 (hydrophobic) in RAD51 points away (Fig. 1d-f). Furthermore, the Loop 2 residues in RAD51, V273 and D274 can form a V273:L238 hydrophobic/steric gate and a D274:R235 salt bridge, which can "lock up" the neighboring triplet basepairs. In DMC1, the hydrophobic/steric effect is smaller and the salt bridge is absent, which can "loosen up" the control for the triplet and allow some conformational flexibility for the basepairs, leading to mismatch tolerance and lower fidelity.

These analyses led us to propose a model (Fig. 1g) where Loop 1 residue Q244 and Loop 2 residues P274/G275 jointly contribute to the mismatch tolerance of DMC1 by stabilizing the DNA backbone and providing extra room and flexibility within the basepair triplet, to accommodate bulkier or staggered mismatched bases. In the following sections, we describe three additional structures and MD simulations to support these structural predictions, followed by functional validation.

Structure of mismatched filaments from DMC1 but not RAD51. Similar to DNA polymerases for which mismatched complexes are often too unstable to be solved, except for lowfidelity polymerases ${ }^{36}$, we found that, with mismatched DNA, DMC1 forms well-ordered filaments, whereas RAD51 forms irregular filaments and two-dimensional (2D) class averages (Supplementary Fig. 5). We then successfully solved the structure of the mismatched dsDNA complex of DMC1 filaments (Structure 3, $3.36 \AA$ ). As the binding is not sequence-specific and as there could only be one mismatch in 15 basepairs (Oligo 3/5 with an A-C mismatch in Supplementary Table 2) for obtaining a stable mismatch structure, the densities of basepairs are averaged out in both homologous and mismatched structures. Nonetheless, the three-dimensional (3D) maps, including DNA backbone and their interacting residues, are essentially identical between the homologous (Structure 2) and mismatched (Structure 3) DMC1 postsynaptic filaments (Supplementary Fig. 6, with a root-meansquared deviation (RMSD) of $0.53 \AA$ out of a total of $309 \mathrm{Ca}$ atoms). These results provide a strong structural support for the mismatch tolerance of DMC1.

Stabilization of DNA by lineage-specific Gln244 and conserved Arg242. We next solved the structure of the postsynaptic filaments of DMC1-Q244M with homologous dsDNA (Structure 4, 
a

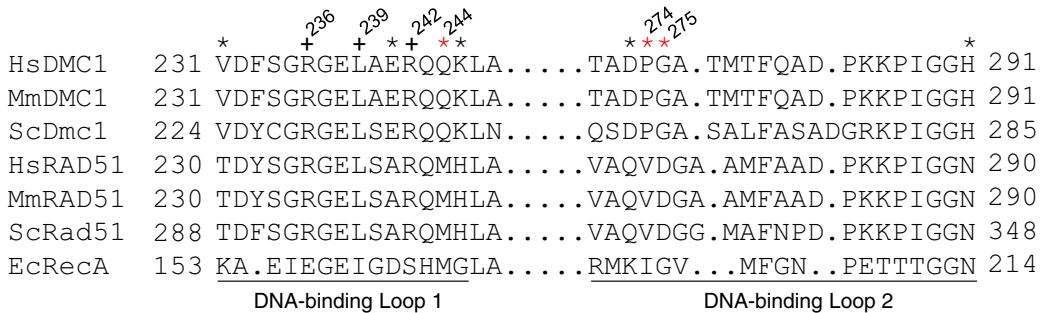

b

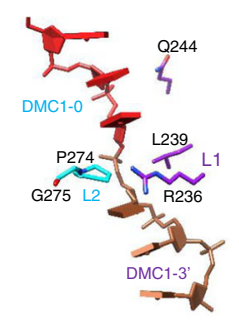

g

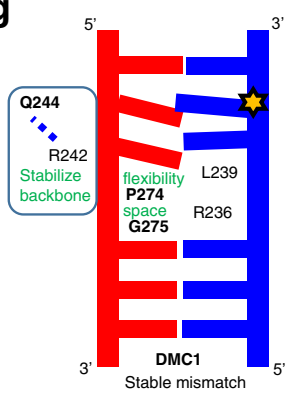

C

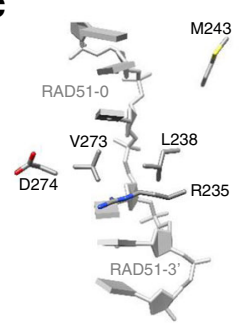

d

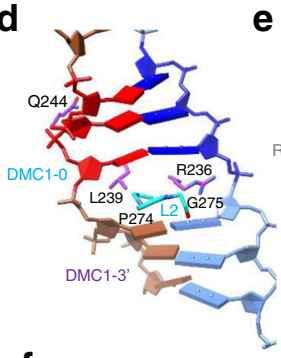

e

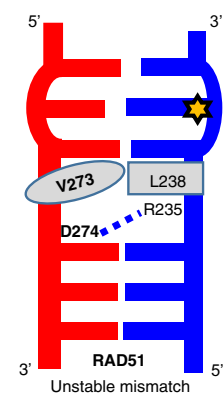

f

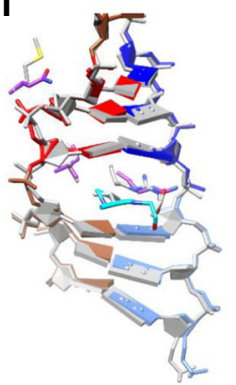

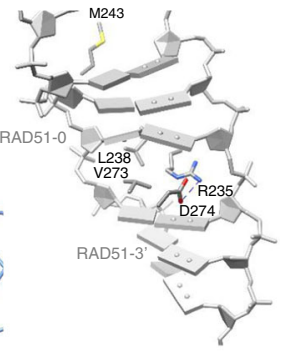

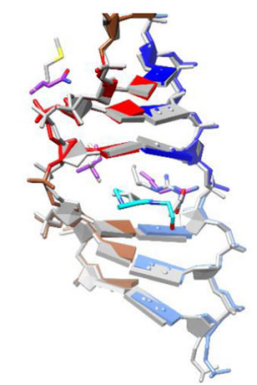

Fig. 1 Possible fidelity-controlling residues of DMC1 and RAD51. a Alignment of the amino acid residues in the Loop 1 and Loop 2 of DNA recombinases from H. sapiens (Hs), M. musculus (Mm), S. cerevisiae (Sc), and E. coli (Ec). All asterisks denote Loop 1 and Loop 2 lineage-specific amino acids, whereas red asterisks denote the three key residues identified in this study. Three conserved residues also addressed in this study are indicated by $\mathbf{a}+$ sign. $\mathbf{b}, \mathbf{c}$ Intertriplet area of DMC1 and RAD51 (PDB 5H1B 28) in the presynaptic filament. $\mathbf{d}$, e The same as $\mathbf{b}, \mathbf{c}$, except for the postsynaptic filaments of DMC1 and RAD51 (PDB 5H1C ${ }^{28}$ ). f Stereo view of the superposed $\mathbf{d}$ and $\mathbf{e}$. The conserved residues DMC1 R236 and L239 from Loop 1, and the lineage-specific residues DMC1 P274 and G275 from Loop 2 are shown in sticks. The labels of DMC1 protomers are based on the designation of RAD51 protomers interacting with a nucleotide triplet from $5^{\prime}$ to $3^{\prime}$ as RAD51-5', RAD51-0, and RAD51-3' as described in Xu et al. ${ }^{28}$. $\mathbf{g}$ A schematic model showing the three lineage-specific residues (bolded) coordinating with three conserved residues (non-bolded) to contribute to the mismatch tolerance (low fidelity) of DMC1 and the unstable mismatch (high fidelity) of RAD51.

$3.20 \AA$ ). Interestingly, the central axis of $2 \mathrm{D}$ class averages, where the DNA is located, is different between DMC1 and DMC1Q244M (Fig. 2a), and only minor residual DNA maps were detected in the $3 \mathrm{D}$ maps of the latter (Fig. 2b). The results suggest that the Q244M mutant still binds DNA for filament formation but the bound DNA is too flexible to be observed by cryo-EM, likely due to the unfavorable replacement of a polar glutamine with a hydrophobic methionine. On the other hand, the protein structure of Q244M filaments is well resolved and is nearly identical to the structure of wild-type (WT) filaments (the backbone RMSD is $0.80 \AA$ out of $305 \mathrm{C}_{\alpha}$ atoms), except that the Met244 sidechain points away from the DNA position (Fig. 2c) similar to that in RAD51 (Fig. 1e). The mismatched dsDNA was also unobservable in its complex with Q244M, but the protein structure could not be solved to a good resolution.

Importantly, as shown in Fig. 2d, the lineage-specific Gln244 appears to coordinate with the conserved Arg242 to stabilize the backbone of DNA in DMC1, with their sidechains spanning three basepairs and reaching out to two non-adjacent phosphodiester moieties. Even though the sidechain of $\operatorname{Gln} 244$ in this duet is somewhat far from the phosphodiester $(4.4 \AA$ for homologous and $4.2 \AA$ for mismatched DNA) for a direct interaction, its functional role in stabilizing DNA backbone cannot be ruled out considering the moderate resolution of the cryo-EM structures and the change of sidechain orientation in the DMC1-Q244M mutant and WT RAD51. Furthermore, due to backbone interactions between Gln244 and Arg242, the sidechain guanidinium group of Arg242 turns away from DNA in Q244M (3.1 $\AA-4.4 \AA$, Fig. 2d). These results suggest that the lineage-specific Gln244 coordinates with the conserved Arg242 to stabilize the backbone of DNA in DMC1, which is weakened in DMC1Q244M, leading to enhanced DNA dynamics and loss of DNA density.

In comparison, such stabilization force is weaker in WT RAD51, with its hydrophobic Met243 pointing away from phosphodiester and Arg242 sidechain $3.5 \AA$ from phosphate (PDB code $5 \mathrm{H}^{2} \mathrm{C}^{28}$ ). With the weakened backbone interaction, RAD51 is less able to stabilize mismatched DNA, as further supported by MD simulations described later.

Increased solvent-accessible volume by Loop 2 P274/G275. To test the hypothesis that DMC1 Loop 2 residues P274/G275 (relative to V273/D274 in RAD51) provide flexibility for DNA basepairs, we further solved the structure of the dsDNA filaments of the double mutant RAD51-VpDg (where the small letters represent corresponding residues from DMC1) (Structure 5, 3.43 $\AA)$. Comparison of the solvent-accessible volume of the cavity (Fig. 3a) shows that the volume around D274 and R235 of RAD51 is cleaved by the salt-bridge interaction, suggesting a rigid environment. In the double mutant RAD51-VpDg, the volume 


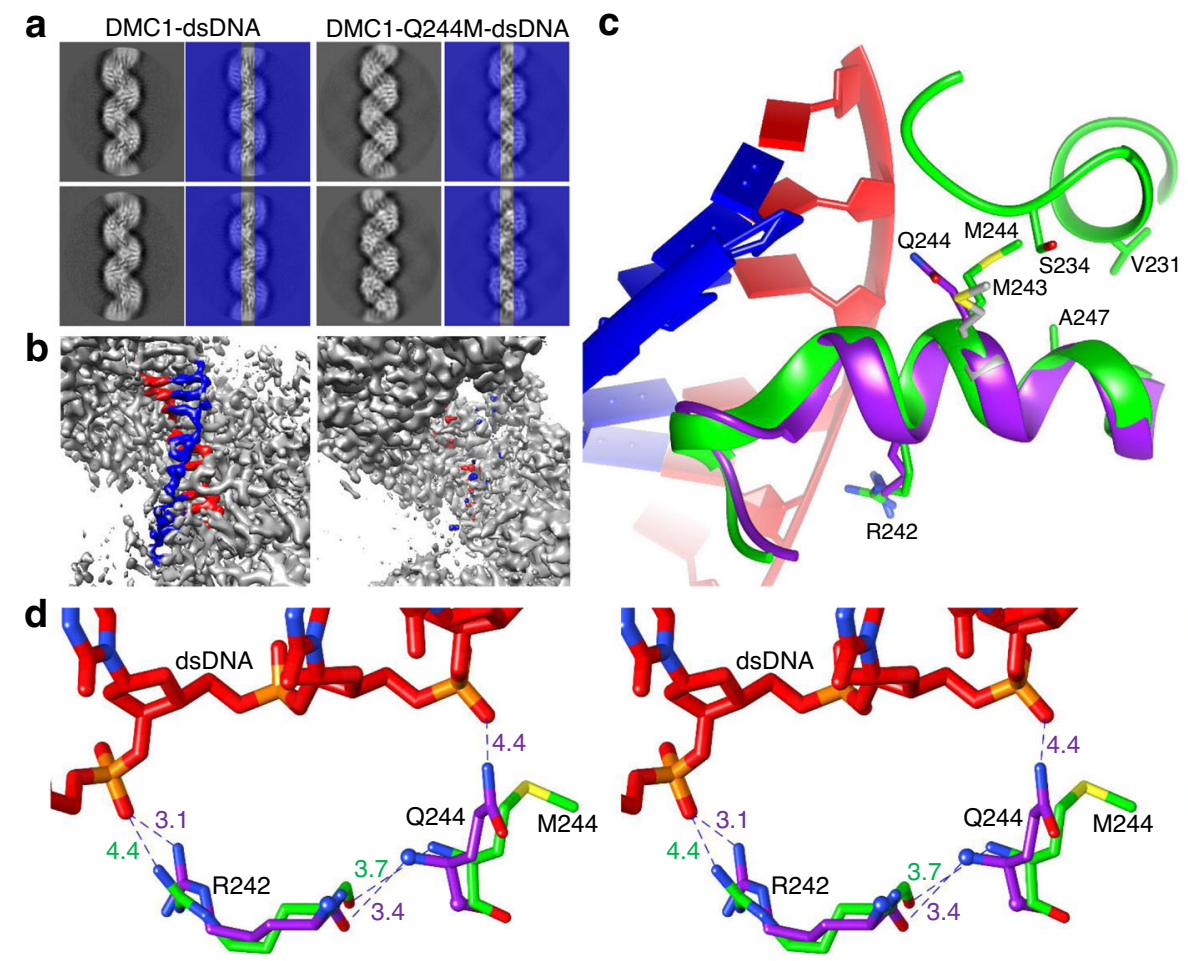

Fig. 2 Structural comparison between the postsynaptic filaments of DMC1 and DMC1-Q244M with homologous dsDNA. a Examples of 2D class average showing DNA in the central axis (highlighted by covering the two sides by blue rectangles with $50 \%$ transparency) of the DMC1 filaments but not the DMC1-Q244M filaments. b Three-dimensional cryo-EM density maps showing that DNA densities (colored in red and blue) can be clearly observed in the DMC1-WT filaments but only residual density can be detected in the DMC1-Q244M filaments. c Superposition of the two structures, showing that the polar sidechain of GIn244 in DMC1 (purple) points toward DNA, whereas that of hydrophobic Met244 in DMC1-Q244M (green) points away from DNA and toward $\mathrm{CH}$ groups of nearby residues. For comparison, Met243 (gray) in RAD51 postsynaptic complex is also shown. $\mathbf{d}$ Stereo view of the relationship between the sidechains of the Gln244-Arg242 pair and the backbone of dsDNA in the postsynaptic filaments of DMC1-WT (purple) and DMC1-Q244M (green). Relevant interatomic distances are shown in $\AA$.

increases again, similar to DMC1. These differences can lead to extra space or flexibility of the triplet boundary to allow for mismatch tolerance by DMC1.

Stabilization of both basepair and backbone by DMC1. We then further performed MD simulations to compare homologous and mismatched structures for the three postsynaptic filaments where the structure with bound dsDNA was available to provide starting coordinates: DMC1, RAD51, and RAD51-VpDg. The RMSD values of the position differences of DNA were calculated throughout the MD simulations to evaluate the mismatch tolerability of each protein, based on their RMSD distributions (Supplementary Fig. 7). As shown in Fig. 3b, the RMSD values of mismatched DNA are substantially larger than those of homologous DNA for RAD51, but only slightly different for DMC1 and RAD51-VpDg, supporting that DMC1 can stabilize mismatched basepairs. Furthermore, plots of the RMSD distributions with DNA backbone only (Fig. 3c) show that the DNA backbone contributes substantially to the RMSD divergence for the mismatched filaments of RAD51 as indicated by the red arrow. In support, the structural ensembles in Fig. 3d show that in the RAD51-mismatch filaments, the DNA backbone, not just the basepairs, is significantly perturbed as highlighted by dashed boxes. The results taken together support that DMC1 can tolerate mismatched dsDNA by stabilizing not only the basepairs (by providing space for misalignment, Fig. 3a) but also the DNA backbone, as proposed in the structural model (Fig. 1g).
Functional activities of DMC1 and RAD51 variants. To validate the roles of Q244, P274, and G275 in the mismatch tolerability of DMC1 based on structural analyses, we performed two functional assays for DMC1, RAD51, and three reciprocal mutants of each: DMC1-Qm, RAD51-Mq, DMC1-PvGd, RAD51-VpDg, DMC1QmPvGd, and RAD51-MqVpDg. For the fidelity comparison to be meaningful, we have ensured that the DNA-binding and strand-exchange activities of the mutants were not substantially perturbed (Supplementary Fig. 8), with the exception of DMC1QmPvGd, which was therefore excluded. The results of all other mutants are summarized in Fig. 4.

First, the mismatch tolerability was examined by a fluorescencebased DNA strand-exchange assay (Fig. 4a, b) ${ }^{37}$. The presynaptic filament was assembled on a ssDNA with a sequence for homologous or mismatched strand exchange. Then, the Cy5 and Cy3 double-labeled dsDNA was added to initiate the strandexchange reaction and the fluorescence signal of $\mathrm{Cy} 3$ was monitored in real-time, which increases as the Cy3-labeled ssDNA is released from the three-strand intermediate, because $\mathrm{Cy} 3$ is no longer close to Cy5. As shown by the time course plots (Fig. 4c) and the bar plots of \% mismatch tolerance (Fig. 4d), compared to the mismatch tolerance of WT human DMC1 at $99.4 \%$, WT human RAD51 displayed 67\%, comparable to its Escherichia coli ortholog RecA (65.4\%, also reported previously ${ }^{29}$ ). The Loop 1 reciprocal mutants DMC1-Qm and RAD51-Mq changed in opposite directions to $77 \%$ and $87 \%$, respectively. Analogously, the Loop 2 reciprocal mutants DMC1-PvGd and RAD51-VpDg changed to $81 \%$ and $100 \%$, respectively. As expected, the triple 
a

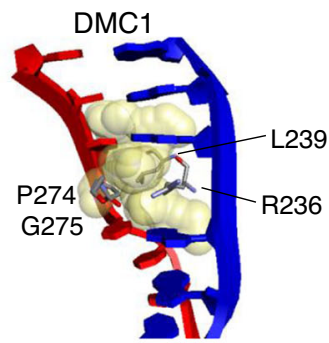

b

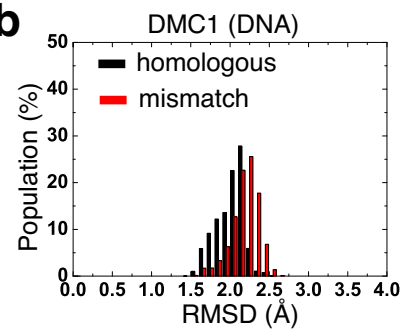

C

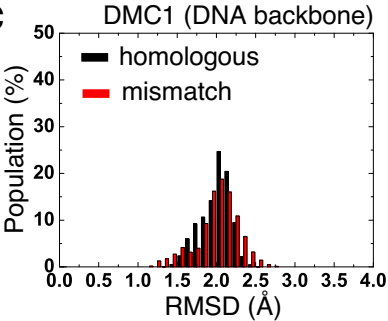

\section{d}
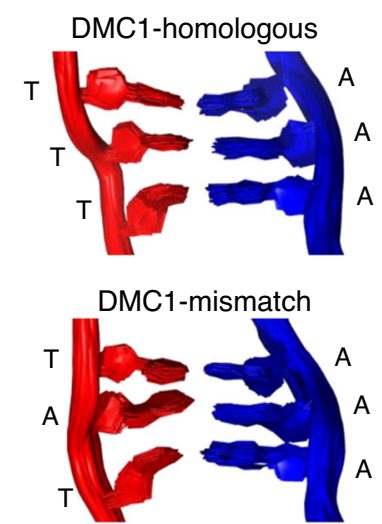
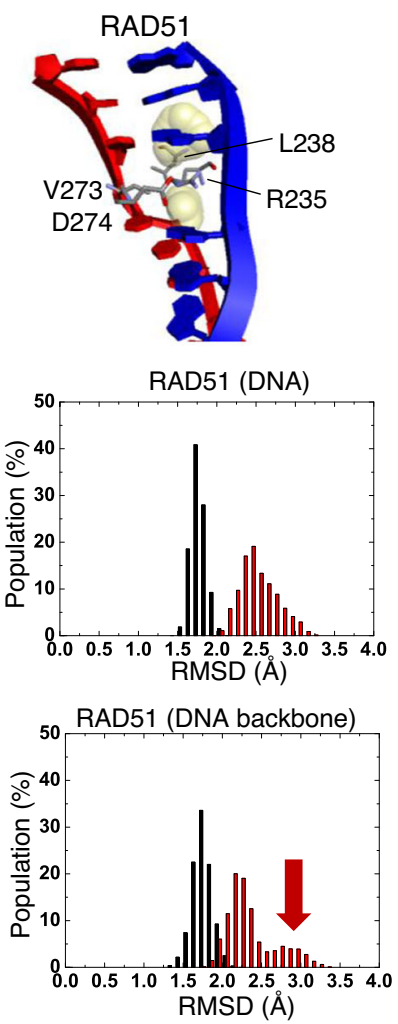

RAD51-homologous

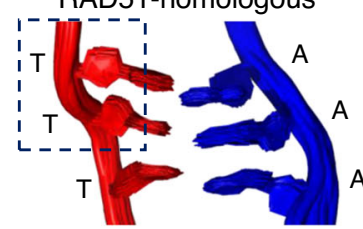

RAD51-mismatch

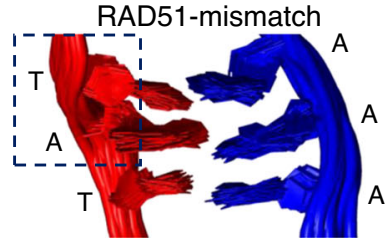

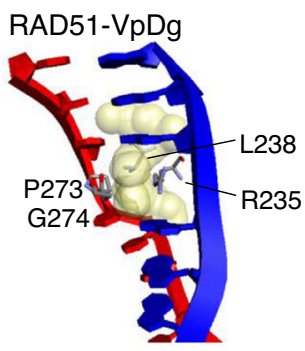
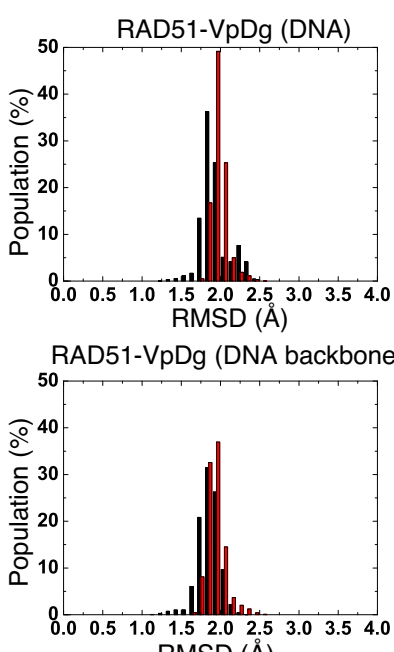

$\operatorname{RMSD}(\AA)$
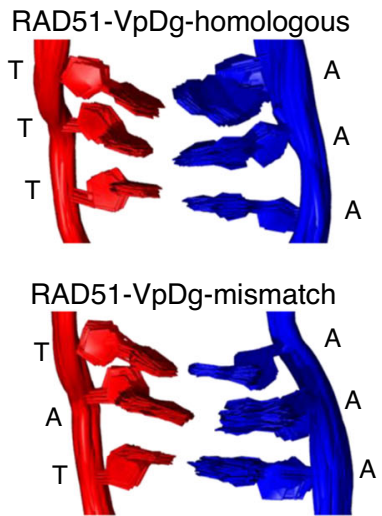

Fig. 3 Flexibility of the basepair triplet in the postsynaptic filaments of DMC1, RAD51, and RAD51-VpDg. a Solvent accessibility within $5 \AA$ around the four gate-keeping residues. The cavities were estimated by point atoms, with radii equal to $1.4 \AA$. The cavity is colored by yellow and the residues are shown as gray stick. b, c RMSD distributions for homologous and mismatched DNA from MD simulations, plotted for all DNA atoms ( 9 basepairs, 574 atoms including hydrogen) (b) and DNA backbone only (106 atoms) (c). The arrow indicates the contribution of DNA backbone to the high-RMSD structures. The overall RMSD fluctuations are shown in Supplementary Fig. 7. d Structural ensembles of the three middle basepairs from MD simulations. The dashed boxes highlight the changes in the DNA backbone between homologous and mismatched RAD51 filaments.

mutant RAD51-MqVpDg also gained $100 \%$ mismatch tolerance activity of DMC1.

We next used single-molecule fluorescence colocalization experiments to monitor the stability of the recombinasemediated three-strand intermediate, the triplex state (Fig. 4e). Surface-anchored and Cy5-labeled DNA substrates containing 81 nt ssDNA were incubated with the recombinase and paired with Cy3-labeled duplex DNA with $15 \mathrm{nt}$ homology, $15 \mathrm{nt}$ containing one mismatch, or $12 \mathrm{nt}$ homology. By monitoring the amount of colocalized $\mathrm{Cy} 3 / \mathrm{Cy} 5$ signal at a given time, we can determine the lifetime of the triplex state and thus the dissociation rate (see plots and values in Fig. 4f). In all panels, the $12 \mathrm{nt}$ homology sample showed the largest dissociation rate, because the short continuous stretch reduces the stability of the triplex state. As expected, although the $15 \mathrm{nt}$ mismatch substrate is closer to the $15 \mathrm{nt}$ homologous substrate than the $12 \mathrm{nt}$ homologous one in WT DMC1, it is closer to the $12 \mathrm{nt}$ homo in WT RAD51. As shown in the other panels in Fig. 4f, both DMC1-Qm and DMC1-PvGd mutants displayed similar properties to WT RAD51, whereas the reciprocal RAD51 mutants as well as the triple mutant RAD51$\mathrm{MqVpDg}$ behaved similar to WT DMC1.

The results from the two different functional assays taken together support that the Loop 1 residue Q244 and the two Loop 2 residues P274/G275 of DMC1, and the corresponding M243/ V273/D274 of RAD51, play key roles in governing the fidelity of both recombinases.

Overall, analyses of five filament structures and MD simulations of WT and mutant recombinases support a model in which DMC1 Loop 2 P274/G275 loosen the gate between basepair triplets to provide space and flexibility for mismatched basepairing, whereas 
Loop 1 Gln244 coordinates with Arg242 to help stabilize the DNA backbone. Although not described in detail, several other conserved residues in Loops 1 and 2 are also intimately involved in DNA binding indirectly, but the three lineage-specific residues addressed here play key roles in the structural differences between DMC1 and
RAD51 filaments. Functional analyses show that mutants of either Loop 1 or Loop 2 can change the fidelity of one recombinase more than half way to the other and the triple mutant RAD51-MqVpDg is as mismatch-tolerant as $\mathrm{DMC1}$, validating the predictions from structural analyses.

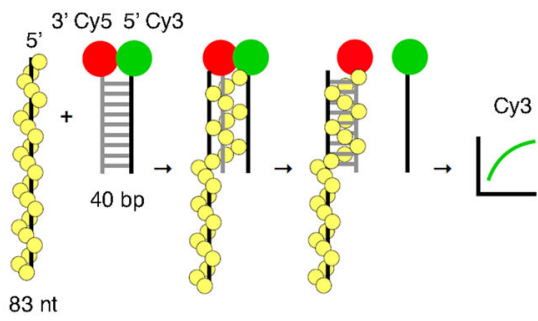

b
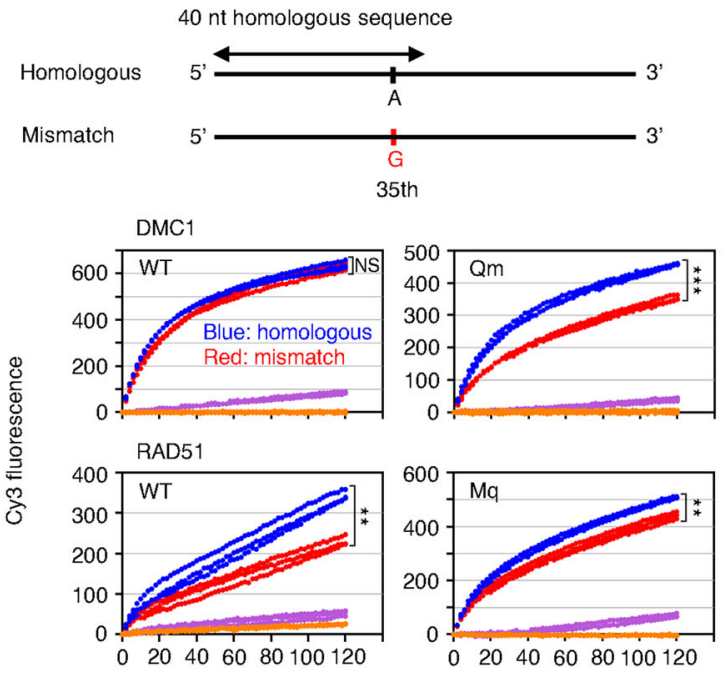

d
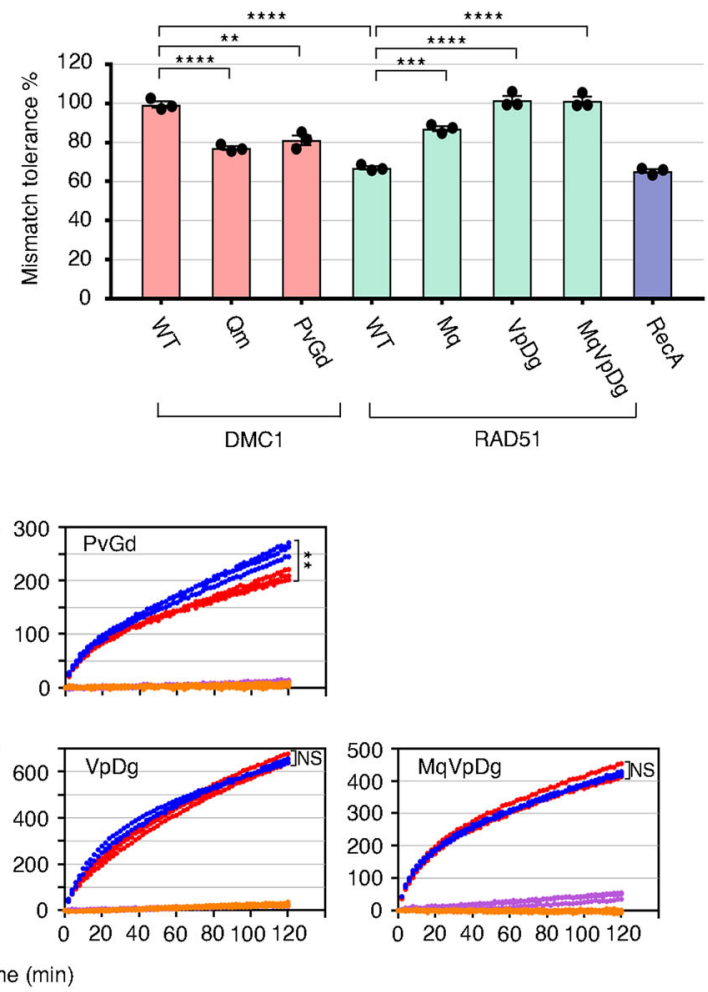

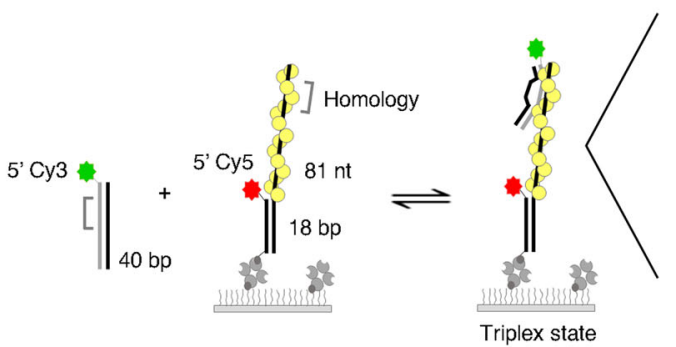

$\mathbf{f}$
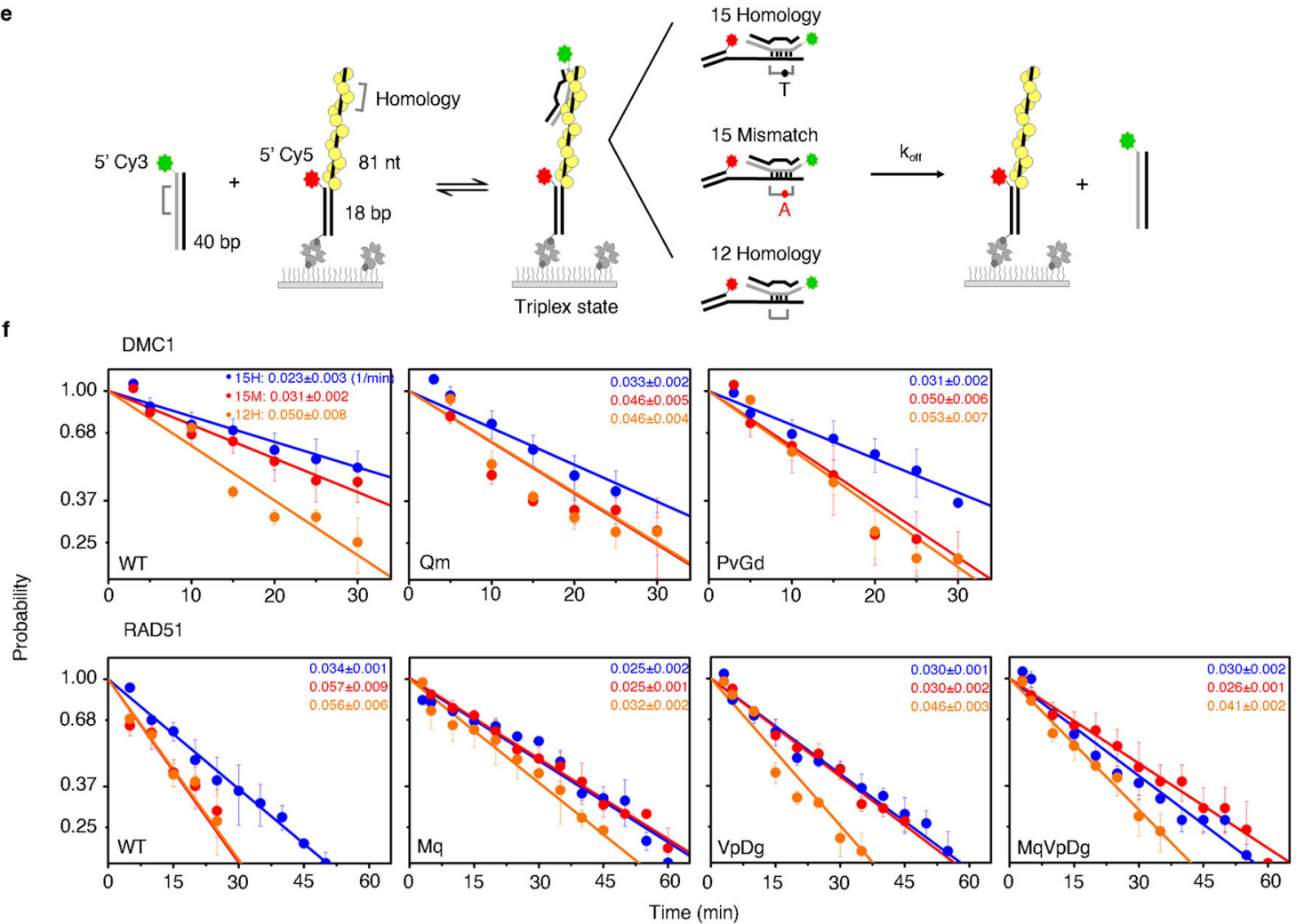
Fig. 4 Functional analysis of the mismatch tolerability of DMC1 and RAD51 variants for Loop 1 and Loop $\mathbf{2}$ residues. a Schematics of fluorescence-based DNA strand exchange. Yellow balls represent the recombinase from filaments on ssDNA. Red and green balls represent Cy5 and Cy3 fluorophores, respectively, at the end of donor dsDNA. b Design diagram of homologous and mismatched substrates. c Cy3 fluorescence signals generated by DMC1, RAD51, and their mutant variants-mediated strand-exchange activity with homologous or mismatch substrates were monitored in real time. Blue and red dots represent the Cy3 fluorescence spectra from the homologous and mismatch substrates, respectively, while purple and orange dots represent the nonhomologous substrate with ATP and homologous substrate without ATP, respectively. Data shown are from three independent experiments. Statistics was performed by two-sides repeated-measure ANOVA (NS, not significant, ${ }^{\star \star} P<0.01$ and ${ }^{\star \star \star} P<0.001$ ). The $P$-values of DMC1-WT, Qm, and PvGd are $0.0757,<0.001$, and 0.005, respectively, whereas for RAD51-WT, Mq, VpDg, and MqVpDg are 0.007, 0.003, 0.224, and 0.806, respectively. d Bar plots for the percentage of mismatch tolerance determined by the final fluorescence intensity of mismatch substrate relative to homologous substrate at 120 min. Data shown are average values (mean) \pm SEM from three independent experiments $(n=3)$. Statistics were performed by one-way ANOVA with Tukey's post hoc test $\left({ }^{\star \star} P<0.01,{ }^{\star \star \star} P<0.001,{ }^{\star \star \star \star} P<0.0001\right)$. The $P$-values of DMC1-WT vs. PvGd and RAD51-WT vs. Mq are 0.0012 and 0.0003 , respectively, whereas that of the rest are $<0.0001$. e Experimental design of single-molecule fluorescence colocalization to determine the stability of the recombinase-mediated triplex state. $\mathbf{f}$ Dissociation of the triplex state as a function of time upon removal of excess duplex strand. Three different DNA substrates ( $15 \mathrm{nt}$ homo, $15 \mathrm{nt}$ mismatch, and $12 \mathrm{nt}$ homo) were determined. Slopes of the plots are the dissociation rates ( $\mathrm{min}^{-1}$ ), which are shown within each panel by blue, red, and orange texts for the $15 \mathrm{nt}$ homo $(15 \mathrm{H}), 15 \mathrm{nt}$ mismatch $(15 \mathrm{M})$, and $12 \mathrm{nt}$ homo $(12 \mathrm{H})$, respectively. Data (mean \pm SEM) are from at least three independent experiments ( $n=3$, except for DMC1-PvGd-12H, $n=4$; RAD51-WT-15M, and 12H, $n=5$ ). Raw data are provided in the Source Data file.

\section{Methods}

Plasmids. The plasmid harboring human $D M C 1$ cDNA in vector pRSFDuet (Novagen) ${ }^{38}$ was subjected to site-directed mutagenesis to construct the DMC1 mutant variants Q244M, PvGd and QmPvGd. The plasmid harboring human RAD51 cDNA in vector pET11 (Novagen) ${ }^{39}$ was subjected to site-directed mutagenesis to construct the RAD51 mutant variants M243Q, VpDg, and MqVpDg. All the resulting plasmids were sequenced to ensure there is no unwanted mutation.

DNA substrates. All oligonucleotides with their sequences are listed in Supplementary Table 2. All oligonucleotides were synthesized and gel purified by Genomics BioSci \& Tech. All of the duplex DNA substrates were prepared by incubating two complementary oligonucleotides in the annealing buffer $(50 \mathrm{mM}$ Tris- $\mathrm{HCl} \mathrm{pH} \mathrm{7.5,} 10 \mathrm{mM} \mathrm{MgCl}_{2}, 100 \mathrm{mM} \mathrm{NaCl}$, and $1 \mathrm{mM}$ dithiothreitol (DTT)) at $80^{\circ} \mathrm{C}$ for $3 \mathrm{~min}$, then at $65^{\circ} \mathrm{C}$ for $30 \mathrm{~min}$, followed by slow cooling to $23^{\circ} \mathrm{C}$ for DNA annealing. The resulting duplex was purified from a $10 \%$ polyacrylamide gel by electro-elution and filter-dialyzed into TE buffer $(10 \mathrm{mM}$ Tris- $\mathrm{HCl} \mathrm{pH} 8.0$ and $0.5 \mathrm{mM}$ EDTA) by a Centricon-10 concentrator (Millipore) at $4{ }^{\circ} \mathrm{C}$.

For cryo-EM, the 80-mer Oligo 1 was used for the assembling of presynaptic filament. The duplex DNA annealed by 15-mer Oligo 3 and Oligo 4 (homologous) or Oligo 3 and Oligo 5 (mismatched) was used for the assembling of postsynaptic filament.

For DNA mobility shift analysis, the Oligo 1 was $5^{\prime}$-end-labeled with $\left[\gamma^{-32} \mathrm{P}\right]$ ATP (PerkinElmer) by polynucleotide kinase (New England Biolabs). Following removal of the unincorporated nucleotide with a Spin 6 column (Bio-Rad), the radiolabeled Oligo 1 was used as the ssDNA substrate. The duplex DNA annealed by radiolabeled Oligo 1 and its exact complement Oligo 2 was used as the dsDNA substrate. For isotope-labeled DNA strand-exchange reactions, the ssDNA Oligo 1 was used to assemble nucleoprotein filament and the $40 \mathrm{bp}$ duplex annealed by Oligo 6 and radiolabeled Oligo 7 was used as the homologous donor dsDNA. For fluorescence-based DNA strand exchange, the 83-mer Oligo 8 (homologous ssDNA), Oligo 9 (mismatched ssDNA), and Oligo 1 (nonhomologous ssDNA) were used to assemble nucleoprotein filament, and the $40 \mathrm{bp}$ duplex annealed by Oligo 10 with $3^{\prime}$-Cy5 fluorophore labeled and Oligo 11 with 5'-Cy3 fluorophore labeled was used as the donor dsDNA.

For single-molecule triplex-state stability experiments, the $3^{\prime}$-biotinylated Cy5labeled hybrid DNA was used for the assembling of presynaptic filament. To prepare the hybrid DNA, 18-mer 3'-biotinylated Oligo 12 with 5'-Cy5 fluorophore labeled was annealed with three different 99 -mer ssDNA, respectively: Oligo 13 containing $15 \mathrm{nt}$ full homology, Oligo 14 containing $15 \mathrm{nt}$ complementary sequence with one mismatch, and Oligo 15 containing $12 \mathrm{nt}$ full homology. Cy3labeled 40 bp dsDNA annealed by Oligo 16 and Oligo 17 was used as the donor dsDNA to form the triplex state.

Protein expression and purification. $\mathrm{His}_{6}$-tagged WT human DMC1 was overexpressed in the E. coli RecA-deficient BLR strain harboring with pRARE to supply tRNAs for rare codons. For purification of the DMC1 protein ${ }^{38}$, the clarified cell lysate was subjected to TALON affinity resin (TaKaRa), Source Q column (GE Healthcare), macrohydroxyapatite column (GE Healthcare), and Mono Q 5/50 GL column (GE Healthcare). The DMC1-containing fractions were pooled, concentrated in a Centricon-30 concentrator (Millipore), and stored in small aliquots at $-80^{\circ} \mathrm{C}$. The $\mathrm{DMC} 1$ variants were expressed and purified as described for the WT protein.

Human RAD51 was expressed in E. coli BLR pRARE strain. The protein purification steps were modified from previously described procedure ${ }^{40}$. Briefly, cell extract was subjected to ammonium sulfate (40\% saturation) precipitation and resuspended in buffer A ( $20 \mathrm{mM} \mathrm{K}_{2} \mathrm{HPO}_{4} \mathrm{pH} 7.5,0.5 \mathrm{mM}$ EDTA, $10 \%$ glycerol, $0.01 \%$ Igepal, $1 \mathrm{mM} \mathrm{2-mercaptoethanol)} \mathrm{supplemented} \mathrm{with} 2 \mathrm{mM}$ Benzamidine, 1 $\mathrm{mM}$ phenylmethylsulfonyl fluoride, and $3 \mu \mathrm{g} / \mathrm{ml}$ of the following protease inhibitors: Aprotinin, Chymostatin, Leupeptin, and Pepstatin A. The RAD51 suspension was then chromatographically fractionated in a Sepharose Q column by applying a $180 \mathrm{ml}$ gradient of $150 \sim 660 \mathrm{mM} \mathrm{KCl}$ in buffer A. The RAD51 pool was diluted with buffer $\mathrm{A}$ and further fractionated in a $1 \mathrm{ml}$ macrohydroxyapatite column using a $90 \mathrm{ml}$ linear gradient of 70 560 $\mathrm{mM} \mathrm{KH}_{2} \mathrm{PO}_{4}$ in buffer A containing $50 \mathrm{mM} \mathrm{KCl}$. The pooled RAD51-containing fraction was diluted and further purified by a $1 \mathrm{ml}$ Source Q column with an $80 \mathrm{ml} 235 \sim 575 \mathrm{mM}$ $\mathrm{KCl}$ gradient in buffer A, and following by a $1 \mathrm{ml}$ Mono Q column with a $45 \mathrm{ml}$ $235 \sim 490 \mathrm{mM} \mathrm{KCl}$ gradient in buffer A. Finally, the RAD51-containing fractions were pooled, concentrated, and stored at $-80^{\circ} \mathrm{C}$. The RAD51 variants were expressed and purified as described for the WT protein. E. coli RecA protein was purchased from New England Biolabs.

Cryo-EM sample preparation and data acquisition. Both presynaptic and postsynaptic complex assembly reaction were incubated with $4 \mu \mathrm{M}$ DMC1 (or RAD51) protein and $24 \mu \mathrm{M}$ nucleotides in buffer B $(35 \mathrm{mM}$ Tris- $\mathrm{HCl} \mathrm{pH} \mathrm{7.5,} 108$ $\mathrm{mM} \mathrm{KCl}$ and $1 \mathrm{mM} \mathrm{DTT}$ ) containing $2 \mathrm{mM} \mathrm{AMP-PNP}$ and $5 \mathrm{mM} \mathrm{CaCl}_{2}$ at $37^{\circ} \mathrm{C}$ for $30 \mathrm{~min}$. Four microliters of protein sample were applied onto a pre-glowdischarged graphene-oxide-coated Quantifoil holey carbon grid (1.2/1.3, 200 mesh) using published protocol ${ }^{41}$. The grids were blotted for $1 \mathrm{~s}$ at $22^{\circ} \mathrm{C}$ with $100 \%$ relative humidity and plunge-frozen in liquid ethane cooled by liquid nitrogen using a Vitrobot Mark IV (Thermo Fisher). Cryo-EM data were acquired on a Titan Krios G3 (Thermo Fisher) microscope operated at $300 \mathrm{keV}$, equipped with a GIF Quantum K2 detector system (Gatan). Automated data acquisition was carried out using EPU software (Thermo Fisher) at a nominal magnification of $\times 165,000$, yielding a pixel size of $0.84 \AA$. Movies of 50 frames, corresponding to a total dose of $50 \mathrm{e}^{-} \AA^{-2}$ were collected in counting mode at a dose rate of $1.0 \mathrm{e}^{-} \AA^{-2}$ per frame.

Image processing and helical reconstruction. The procedures are summarized in Supplementary Fig. 1. All movies were motion-corrected and dose-weighted using RELION's own implementation ${ }^{42}$. Only aligned, non-dose-weighted micrographs were then used to estimate the contrast transfer function with Gctf ${ }^{43}$. All subsequent image-processing steps were carried out using helical reconstruction in RELION $3.0^{44}$. Filaments were manually picked and segments were extracted using a box size of 384 pixels and an inter-box distance of $\sim 10 \%$ of the box length. A spherical mask with a diameter equal to $90 \%$ of the box size was applied. Iterative rounds of $2 \mathrm{D}$ classification were performed to remove low-quality filaments. A simple cylinder was used as initial model to prevent model bias. Together with the selected particles, 3D classification was performed using the helical rise and twist derived from RAD51 presynaptic filaments as initial helical parameters 28,35 . The reconstruction served as an initial reference model for 3D auto-refinement. All refinements were carried out following the gold-standard procedure where the data set was divided into two half-sets. After refinement was converged, the corrected Fourier shell correlation $(0.143 \AA)$ criterion was calculated to estimate the resolution after post-processing with a soft mask applied.

Model building and refinement. The initial atomic model of human DMC1 protomers in both the presynaptic and postsynaptic complexes was generated from homology modeling of the cryo-EM structure of human RAD51 filaments ${ }^{28,45}$. The structures were then docked into the EM map by using PHENIX ${ }^{46}$. DNA and AMP-PNP were manually appended in $\mathrm{COOT}^{47}$ and rigid-body docked into the electron density map in UCSF-Chimera ${ }^{48}$. The built models were replicated 
multiple times and fitted into the EM maps of the presynaptic and postsynaptic complexes in UCSF-Chimera by following their corresponding helical symmetries to generate the atomic models of longer assemblies. The models of presynaptic and postsynaptic complexes were further refined in Rosetta ${ }^{49}$. The refined atomic models were further validated and manually inspected using PHENIX ${ }^{46}$ and $\mathrm{COOT}^{47}$. Since the binding is not sequence-specific and the densities for the bases are averages, they were fitted with poly-dT (ssDNA) and poly-dT:poly-dA (dsDNA, invading strand and complementary strand, respectively) for model building.

MD simulations. MD simulations in explicit water were conducted using the Amber 16 package ${ }^{50}$. For DMC1 and RAD51-VpDg, our cryo-EM models (consisting of three protein protomers and nine A-T basepairs) provided the starting protein coordinates. For RAD51, the initial structure was based on PDB $5 \mathrm{H}_{1} \mathrm{C}^{28}$. All single mismatch-containing DNA was rebuilt by Web 3DNA $2.0^{51}$, where the Thymine in the middle position was mutated to Adenine. The simulations were performed using Amber ff14SB force field ${ }^{52}$ and the residue charges were calculated based on the libraries in the Amber 16 package. Periodic boundary conditions were imposed with box lengths of $107.5 \times 95.1 \times 121.8 \AA^{3}$. The atoms of some loop 1 and loop 2 residues, e.g., R236, P274, G275, and Q244 of DMC1 were constrained. A Langevin thermostat was used to maintain the system temperature with collision frequency of $1 \mathrm{ps}^{-1}$ to the target temperature $300 \mathrm{~K}$. The SHAKE algorithm was implemented to constrain the covalent bond. All MD simulations were carried out in the isothermal-isobaric ensemble with the Langevin thermostat and the time step of $1 \mathrm{fs}$. The system first underwent an annealing process from 0 to $300 \mathrm{~K}$ under a constant pressure of 1.0 bar over $7 \mathrm{~ns}$ and maintained at this equilibrated point for the following simulation. After equilibrated steps, the final equilibrium system density was $\sim 1.0 \pm 0.01 \mathrm{~g} / \mathrm{cm}^{3}$. Finally, we further performed $13 \mathrm{~ns}$ MD simulations for checking systems equilibrium and RMSD analysis.

Electrophoretic mobility shift assay. The ${ }^{32} \mathrm{P}$-labeled 80 -mer ssDNA $(3 \mu \mathrm{M}$ nucleotides) and ${ }^{32} \mathrm{P}$-labeled $80 \mathrm{bp}$ dsDNA ( $3 \mu \mathrm{M}$ basepairs) were incubated individually with the indicated amount of human DMC1 or RAD51 variants in 10 $\mu \mathrm{l}$ of buffer C (35 mM Tris- $\mathrm{HCl}$ pH 7.5, $1 \mathrm{mM}$ DTT, $10 \mathrm{mM} \mathrm{MgCl}_{2}, 100 \mathrm{ng} / \mu \mathrm{l}$ bovine serum albumin (BSA), $50 \mathrm{mM} \mathrm{KCl}$ and $2 \mathrm{mM} \mathrm{ATP}$ ) at $37^{\circ} \mathrm{C}$ for $10 \mathrm{~min}$. The reaction mixtures were fractionated in $2 \%$ agarose gels in TAE buffer $(40 \mathrm{mM}$ Tris, $20 \mathrm{mM}$ acetate $\mathrm{pH} 7.5$, and $2 \mathrm{mM} \mathrm{EDTA}$ ) at $4{ }^{\circ} \mathrm{C}$. The gels were dried onto DE81 paper and subjected to phosphorimaging analysis (Bio-Rad Personal Molecular Imager) and quantitative analysis (Bio-Rad Quantity One 4.6.9).

DNA strand-exchange assay. The 80 -mer Oligo $1(4.8 \mu \mathrm{M}$ nucleotides) was preincubated with the indicated amount of human DMC1 or RAD51 variants individually to assemble nucleoprotein filament in buffer C supplemented with $10 \mathrm{mM}$ $\mathrm{CaCl}_{2}$ at $37^{\circ} \mathrm{C}$ for $5 \mathrm{~min}$. The reaction was initiated by adding homologous ${ }^{32} \mathrm{P}-$ labeled 40 -mer duplex ( $2.4 \mu \mathrm{M}$ basepairs) to a final volume of $12.5 \mu \mathrm{l}$. After a 30 min incubation, a $5 \mu \mathrm{l}$ aliquot was removed, mixed with an equal volume of $0.1 \%$ SDS containing proteinase $\mathrm{K}(1 \mathrm{mg} / \mathrm{ml})$, and incubated at $37^{\circ} \mathrm{C}$ for $15 \mathrm{~min}$. The samples were fractionated in $10 \%$ polyacrylamide gels in TBE buffer $(89 \mathrm{mM}$ Tris, $89 \mathrm{mM}$ borate, and $2 \mathrm{mM}$ EDTA pH 8.0). Gels were dried onto DE81 paper and subjected to phosphorimaging and quantitative analysis.

Fluorescence-based DNA strand exchange. The 83-mer Oligo 8, Oligo 9, or 80mer Oligo 1 ( $3 \mu \mathrm{M}$ nucleotides) harboring the homologous, one mismatched, or nonhomologous sequence, respectively, was incubated with human DMC1 variants $(1 \mu \mathrm{M}$ for WT and Qm mutant, and $2 \mu \mathrm{M}$ for PvGd mutant) or RAD51 variants $(0.75 \mu \mathrm{M})$ to assemble nucleoprotein filament in $40 \mu \mathrm{l}$ buffer $\mathrm{C}$ supplemented with $10 \mathrm{mM} \mathrm{CaCl}_{2}$. After $30 \mathrm{~min}$ incubation on ice, $10 \mu \mathrm{l} \mathrm{Cy} 5$ and Cy3 double-labeled dsDNA ( $1.45 \mu \mathrm{M}$ basepairs) were added to initiate the strand-exchange reaction The reaction mixture was transferred into Falcon 384-Well Optilux Black/Clear Flat and the fluorescence emission of Cy3 at $590 \mathrm{~nm}$ (bandwidth: $35 \mathrm{~nm}$ ) upon excitation at $530 \mathrm{~nm}$ (bandwidth: $25 \mathrm{~nm}$ ) was monitored in real-time by Gene5 2.07 spectrofluorometer (BioTek SynergyHTX multi-mode reader). Data were collected every two min at room temperature. The Cy3 emission signal $\left(F_{t}\right)$ representing the change in the amount of strand exchange product at each time point was calculated using following equation: $F_{\mathrm{t}}=\left(S_{\mathrm{t}}-S_{0}\right)-\left(B_{\mathrm{t}}-B_{0}\right)$. " $\mathrm{S}_{\mathrm{t}}$ " and " $S_{0}$ " are the fluorescence intensity from the reaction containing protein at each time point or beginning, respectively, whereas " $B_{\mathrm{t}}$ " and " $B_{0}$ " are from the reaction without protein. The fluorescence spectra of $\mathrm{Cy} 3$ signals were plotted by the time correlated to $F_{\mathrm{t}}$. The relative strand-exchange efficiency $\left(R_{\mathrm{f}}\right.$ shown in \%) for the calculation of mismatch tolerability of recombinase is calculated with the following equation: $R_{\mathrm{f}}=F_{\mathrm{M}} / F_{\mathrm{H}}$, where " $F_{\mathrm{M}}$ " is the Cy3 emission signal $\left(F_{\mathrm{t}}\right)$ at the $120 \mathrm{~min}$ time point of the reaction with mismatched substrates and " $F_{\mathrm{H}}$ " is the Cy3 emission signal of the reaction with homologous substrates at the $120 \mathrm{~min}$ time point.

Single-molecule triplex-state stability experiments. The reaction chamber was constructed by incubating $0.02 \mathrm{mg} / \mathrm{mL}$ streptavidin for $5 \mathrm{~min}$ in the PEGylated slide and coverslip ${ }^{53}$. After washing excess streptavidin with buffer D (20 mM Tris$\mathrm{HCl}$ and $50 \mathrm{mM} \mathrm{NaCl}$ ), three different $3^{\prime}$-biotinylated Cy5-labeled hybrid DNA containing $15 \mathrm{nt}$ full homology, $15 \mathrm{nt}$ with one mismatch, and 12nt full homology, individually, were immobilized on the surface for $5 \mathrm{~min}$. It was then washed with buffer E (buffer F: $35 \mathrm{mM}$ Tris-HCl pH 7.5, $1 \mathrm{mM}$ DTT, $10 \mathrm{mM} \mathrm{MgCl} 2,1 \mu \mathrm{g} / \mu \mathrm{l}$ $\mathrm{BSA}, 50 \mathrm{mM} \mathrm{KCl}$, and $10 \mathrm{mM} \mathrm{CaCl}$; supplementing with $4 \mathrm{mg} / \mathrm{ml}$ glucose, $30 \mathrm{U} /$ $\mathrm{ml}$ glucose oxidase, and $30 \mathrm{U} / \mathrm{ml}$ catalase). Recombinase (1 $\mu \mathrm{M} \mathrm{RAD} 51 / \mathrm{mutant}$ or $2 \mu \mathrm{M} \mathrm{DMC1}$ /mutant) in buffer F supplemented with $2 \mathrm{mM}$ ATP was then incubated with surface-bound hybrid DNA for $15 \mathrm{~min}$ to form nucleoprotein filaments. Triplex-state formation was carried out by washing excess recombinases and flowing $10 \mathrm{nM}$ Cy3-labeled $40 \mathrm{bp}$ dsDNA into the reaction chamber for $20 \mathrm{~min}$. Free Cy3-labeled $40 \mathrm{bp}$ dsDNA was then washed three times using imaging buffer $\mathrm{E}$ with $2 \mathrm{mM}$ ATP but without $\mathrm{CaCl}_{2}$ and it was defined as time zero. The numbers of triplex state were monitored at given time points by scoring the numbers of colocalized Cy3/Cy5 spots in the same field-of-view. At each time point, 20 frames $(2 \mathrm{~s})$ were recorded sequentially with green and red lasers to image Cy3 and Cy5 dyes, respectively.

We utilized objective-type total internal reflection fluorescence microscope (IX71; Olympus) equipped with 532 and $633 \mathrm{~nm}$ lasers to alternatively excite Cy3 and Cy5. The fluorescence signal was acquired by an electron-multiplying CCD (ProEM 512B; Princeton Instruments) at $10 \mathrm{~Hz}$ using a dual-view system. The image recorded using a software program is written in LABVIEW 8.6. Data analysis was performed using MATLAB for automatic spot detection and colocalization. The software used for fitting was Origin.

Statistical analysis. The difference between experimental curve with homologous and mismatched substrates for each protein was analyzed with two-sides repeatedmeasures analysis of variance (ANOVA). Between-subject effects were determined by fluorescent data extracted from every $10 \mathrm{~min}$ and calculated with Statistical Product and Service Solutions. $P$-value $<0.05$ indicates that the reaction is significantly different between homologous and mismatched substrates. The significance between mismatch tolerance of WT and mutant variant protein was analyzed with GraphPad Prism 7. Multiple groups were compared using one-way ANOVA with Tukey's post hoc test. $P$-values are represented as $P>0.05$ is not significant and $P<0.05$ is significant. The normality of data was tested by Shapiro-Wilk normality test to confirm that the data was normally distributed.

Reporting summary. Further information on the research design can be found in the Nature Research Reporting Summary linked to this paper.

\section{Data availability}

The refined coordinates and corresponding cryo-EM maps have been deposited in the Protein Data Bank and the Electron Microscopy Data Bank under accession codes "PDB7C9C" and EMD-30311 (DMC1 presynaptic complex), "PDB-7C98" and EMD-30308 (DMC1 postsynaptic complex), "PDB-7C99" and EMD-30309 (DMC1 postsynaptic complex with mismatch), "PDB-7CGY" and EMD-30366 (DMC1-Q244M mutant postsynaptic complex), "PDB-7C9A" and EMD-30310 (RAD51-V273P, D274G mutant postsynaptic complex). All other relevant data are described in the Supplementary Information. Any additional data related to this paper are available upon request. Source data are provided with this paper.

Received: 14 September 2020; Accepted: 23 November 2020; Published online: 14 January 2021

\section{References}

1. Moynahan, M. E. \& Jasin, M. Mitotic homologous recombination maintains genomic stability and suppresses tumorigenesis. Nat. Rev. Mol. Cell Biol. 11, 196-207 (2010).

2. Symington, L. S., Rothstein, R. \& Lisby, M. Mechanisms and regulation of mitotic recombination in Saccharomyces cerevisiae. Genetics 198, 795-835 (2014).

3. Kowalczykowski, S. C. An overview of the molecular mechanisms of recombinational DNA repair. Cold Spring Harb. Perspect. Biol. 7, a016410 (2015).

4. Haber, J. E. A life investigating pathways that repair broken chromosomes. Annu. Rev. Genet. 50, 1-28 (2016).

5. Cox, M. M. et al. The importance of repairing stalled replication forks. Nature 404, 37-41 (2000).

6. Sehorn, M. G. \& Sung, P. Meiotic recombination: an affair of two recombinases. Cell Cycle 3, 1375-1377 (2004).

7. Neale, M. J. \& Keeney, S. Clarifying the mechanics of DNA strand exchange in meiotic recombination. Nature 442, 153-158 (2006).

8. Brown, M. S. \& Bishop, D. K. DNA strand exchange and RecA homologs in meiosis. Cold Spring Harb. Perspect. Biol. 7, a016659 (2014).

9. San Filippo, J., Sung, P. \& Klein, H. Mechanism of eukaryotic homologous recombination. Annu. Rev. Biochem. 77, 229-257 (2008). 
10. Heyer, W. D., Ehmsen, K. T. \& Liu, J. Regulation of homologous recombination in eukaryotes. Annu. Rev. Genet. 44, 113-139 (2010).

11. Qi, Z. et al. DNA sequence alignment by microhomology sampling during homologous recombination. Cell 160, 856-869 (2015).

12. Greene, E. C. DNA sequence alignment during homologous recombination. J. Biol. Chem. 291, 11572-11580 (2016).

13. Bishop, D. K., Park, D., Xu, L. \& Kleckner, N. DMC1: a meiosis-specific yeast homolog of E. coli recA required for recombination, synaptonemal complex formation, and cell cycle progression. Cell 69, 439-456 (1992).

14. Lao, J. P. et al. Meiotic crossover control by concerted action of Rad51-Dmc1 in homolog template bias and robust homeostatic regulation. PLoS Genet. 9, e1003978 (2013).

15. Callender, T. L. et al. Mek1 down regulates Rad51 activity during yeast meiosis by phosphorylation of Hed1. PLoS Genet. 12, e1006226 (2016).

16. Crickard, J. B. \& Greene, E. C. The biochemistry of early meiotic recombination intermediates. Cell Cycle 17, 2520-2530 (2018).

17. Steinfeld, J. B. et al. Defining the influence of Rad51 and Dmc1 lineagespecific amino acids on genetic recombination. Genes Dev. 33, 1191-1207 (2019).

18. Ogawa, T., Yu, X., Shinohara, A. \& Egelman, E. H. Similarity of the yeast RAD51 filament to the bacterial RecA filament. Science 259, 1896-1899 (1993).

19. Masson, J. Y. \& West, S. C. The Rad51 and Dmc1 recombinases: a nonidentical twin relationship. Trends Biochem. Sci. 26, 131-136 (2001).

20. Lin, Z., Kong, H., Nei, M. \& Ma, H. Origins and evolution of the recA/RAD51 gene family: evidence for ancient gene duplication and endosymbiotic gene transfer. Proc. Natl Acad. Sci. USA 103, 10328-10333 (2006).

21. Crickard, J. B. \& Greene, E. C. Biochemical attributes of mitotic and meiotic presynaptic complexes. DNA Repair (Amst.) 71, 148-157 (2018).

22. Egelman, E. H. \& Stasiak, A. Structure of helical RecA-DNA complexes. Complexes formed in the presence of ATP-gamma-S or ATP. J. Mol. Biol. 191, 677-697 (1986).

23. Sung, P. Catalysis of ATP-dependent homologous DNA pairing and strand exchange by yeast RAD51 protein. Science 265, 1241-1243 (1994).

24. Sehorn, M. G., Sigurdsson, S., Bussen, W., Unger, V. M. \& Sung, P. Human meiotic recombinase Dmcl promotes ATP-dependent homologous DNA strand exchange. Nature 429, 433-437 (2004).

25. Sheridan, S. D. et al. A comparative analysis of Dmcl and Rad51 nucleoprotein filaments. Nucleic Acids Res. 36, 4057-4066 (2008)

26. Chen, Z., Yang, H. \& Pavletich, N. P. Mechanism of homologous recombination from the RecA-ssDNA/dsDNA structures. Nature 453, 489-484 (2008).

27. Lee, J. Y. et al. DNA RECOMBINATION. Base triplet stepping by the Rad51/ RecA family of recombinases. Science 349, 977-981 (2015).

28. $\mathrm{Xu}$, J. et al. Cryo-EM structures of human RAD51 recombinase filaments during catalysis of DNA-strand exchange. Nat. Struct. Mol. Biol. 24, 40-46 (2017).

29. Borgogno, M. V. et al. Tolerance of DNA mismatches in Dmc1 recombinase-mediated DNA strand exchange. J. Biol. Chem. 291, 4928-4938 (2016).

30. Lee, J. Y. et al. Sequence imperfections and base triplet recognition by the Rad51/RecA family of recombinases. J. Biol. Chem. 292, 11125-11135 (2017).

31. Story, R. M., Bishop, D. K., Kleckner, N. \& Steitz, T. A. Structural relationship of bacterial RecA proteins to recombination proteins from bacteriophage T4 and yeast. Science 259, 1892-1896 (1993).

32. Kinebuchi, T. et al. Structural basis for octameric ring formation and DNA interaction of the human homologous-pairing protein Dmcl. Mol. Cell 14, 363-374 (2004).

33. Conway, A. B. et al. Crystal structure of a Rad51 filament. Nat. Struct. Mol. Biol. 11, 791-796 (2004).

34. Prentiss, M., Prevost, C. \& Danilowicz, C. Structure/function relationships in RecA protein-mediated homology recognition and strand exchange. Crit. Rev. Biochem. Mol. Biol. 50, 453-476 (2015).

35. Short, J. M. et al. High-resolution structure of the presynaptic RAD51 filament on single-stranded DNA by electron cryo-microscopy. Nucleic Acids Res. 44, 9017-9030 (2016).

36. Wu, W.-J., Yang, W. \& Tsai, M.-D. How DNA polymerases catalyse replication and repair with contrasting fidelity. Nat. Rev. Chem. 1, 0068 (2017).

37. Ito, K., Argunhan, B., Tsubouchi, H. \& Iwasaki, H. Real-time observation of the DNA strand exchange reaction mediated by Rad51. JoVE e59073 (2019).

38. Chang, H. Y. et al. Functional relationship of ATP hydrolysis, presynaptic filament stability, and homologous DNA pairing activity of the human meiotic recombinase DMC1. J. Biol. Chem. 290, 19863-19873 (2015).
39. Sigurdsson, S., Trujillo, K., Song, B., Stratton, S. \& Sung, P. Basis for avid homologous DNA strand exchange by human Rad51 and RPA. J. Biol. Chem. 276, 8798-8806 (2001).

40. Chi, P., Van Komen, S., Sehorn, M. G., Sigurdsson, S. \& Sung, P. Roles of ATP binding and ATP hydrolysis in human Rad51 recombinase function. DNA Repair 5, 381-391 (2006).

41. Palovcak, E. et al. A simple and robust procedure for preparing grapheneoxide cryo-EM grids. J. Struct. Biol. 204, 80-84 (2018).

42. Scheres, S. H. RELION: implementation of a Bayesian approach to cryo-EM structure determination. J. Struct. Biol. 180, 519-530 (2012).

43. Zhang, K. Gctf: real-time CTF determination and correction. J. Struct. Biol. 193, 1-12 (2016).

44. He, S. \& Scheres, S. H. W. Helical reconstruction in RELION. J. Struct. Biol. 198, 163-176 (2017).

45. Guex, N., Peitsch, M. C. \& Schwede, T. Automated comparative protein structure modeling with SWISS-MODEL and Swiss-PdbViewer: a historical perspective. Electrophoresis 30(Suppl 1), S162-S173 (2009).

46. Afonine, P. V. et al. Real-space refinement in PHENIX for cryo-EM and crystallography. Acta Crystallogr. D Struct. Biol. 74, 531-544 (2018).

47. Emsley, P., Lohkamp, B., Scott, W. G. \& Cowtan, K. Features and development of Coot. Acta Crystallogr. D Biol. Crystallogr 66, 486-501 (2010).

48. Pettersen, E. F. et al. UCSF Chimera-a visualization system for exploratory research and analysis. J. Comput. Chem. 25, 1605-1612 (2004).

49. Wang, R. Y.-R. et al. Automated structure refinement of macromolecular assemblies from cryo-EM maps using Rosetta. eLife 5, e17219 (2016).

50. Case, D. A. et al. AMBER 16 (Univ. California, San Francisco, 2016).

51. Li, S., Olson, W. K. \& Lu, X. J. Web 3DNA 2.0 for the analysis, visualization, and modeling of 3D nucleic acid structures. Nucleic Acids Res. 47, W26-W34 (2019).

52. Maier, J. A. et al. ff14SB: improving the accuracy of protein side chain and backbone parameters from ff99SB. J. Chem. Theory Comput 11, 3696-3713 (2015).

53. Lu, C. H. et al. Swi5-Sfr1 stimulates Rad51 recombinase filament assembly by modulating Rad51 dissociation. Proc. Natl Acad. Sci. USA 115 E10059-E10068 (2018).

\section{Acknowledgements}

This work was supported by Academia Sinica (M.-D.T., P.C., and M.-C.H.), National Taiwan University (P.C. and H.W.L.), Taiwan Ministry of Science and Technology (MOST 105-2314-B-002-073-MY4 and MOST 108-2321-B-002-054 to P.C., MOST-107 2113-M-002-010-MY3 to H.-W.L.), and Taiwan Protein Project (Grant number ASKPQ-105-TPP and AS-KPQ-109-TPP2 to M.-D.T.). The cryo-EM experiments were performed at the Academia Sinica Cryo-EM Center (ASCEM) and the cryo-EM data were processed at the Academia Sinica Grid-computing Center (ASGC). ASCEM is supported by Academia Sinica (Grant number AS-CFII-108-110) and Taiwan Protein Project. ASGC is supported by Academia Sinica.

\section{Author contributions}

P.C., M.-C.H., and M.-D.T. conceived and designed the research. S.-C.L., Y.-M.W., and M.-C.H. performed cryo-EM experiments and processed data. H.-Y.Y., H.-Y.C., G.-C.S., C.-Y.L., and P.C. prepared proteins and designed and performed functional assays. W.-H.L. and H.-W.L. designed and performed single-molecule assays. C.-H.Y. performed computational analysis. All authors analyzed data. M.-D.T., P.C., and M.-C.H. supervised the project and interpret overall data. S.-C.L., H.-Y.Y., W.-J.W., M.-C.H., P.C., and M.-D.T. wrote the paper.

\section{Competing interests}

The authors declare no competing interests.

\section{Additional information}

Supplementary information is available for this paper at https://doi.org/10.1038/s41467 020-20258-1.

Correspondence and requests for materials should be addressed to M.-C.H., P.C. or M.D.T.

Peer review information Nature Communications thanks the anonymous reviewer(s) for their contribution to the peer review of this work. Peer reviewer reports are available.

Reprints and permission information is available at http://www.nature.com/reprints

Publisher's note Springer Nature remains neutral with regard to jurisdictional claims in published maps and institutional affiliations. 
(c) (i) Open Access This article is licensed under a Creative Commons Attribution 4.0 International License, which permits use, sharing, adaptation, distribution and reproduction in any medium or format, as long as you give appropriate credit to the original author(s) and the source, provide a link to the Creative Commons license, and indicate if changes were made. The images or other third party material in this article are included in the article's Creative Commons license, unless indicated otherwise in a credit line to the material. If material is not included in the article's Creative Commons license and your intended use is not permitted by statutory regulation or exceeds the permitted use, you will need to obtain permission directly from the copyright holder. To view a copy of this license, visit http://creativecommons.org/ licenses/by/4.0/.

(C) The Author(s) 2021 